\title{
Evaluation of a Critical Sized Bone Defect in a Porcine Tibial Model
}

\author{
Anthony J. Milto ${ }^{1}$, Alexander W. Peters ${ }^{1}$, Aamir Tucker ${ }^{1}$, Alex Brinker ${ }^{1}$, Michael \\ Savaglio ${ }^{1}$, Gremah Adam ${ }^{1}$, Venkateswaran Ganesh ${ }^{1}$, Zachary Gunderson ${ }^{1}$, Paul \\ Childress $^{1}$, Roman M. Natoli ${ }^{1}$, Todd O. McKinley ${ }^{1}$, Melissa A. Kacena ${ }^{1}$, \\ ${ }^{1}$ Department of Orthopaedic Surgery, Indiana University School of Medicine, \\ Indianapolis, IN, USA
}

\section{Background/Hypothesis: \\ Few large animal bone injury models exist. We present a porcine tibial model of metacritical and critical sized bone defects for the simulation of traumatic bone injuries.}

\section{Project Methods:}

16 pigs were used in this study. The pigs were divided into 3 groups $(n=4-$ 8/group). In 12 pigs, a $25 \mathrm{~mm}$ osteotomy was created in the tibia and the space was filled with a $25 \mathrm{~mm}$ scaffold. 8 of the $25 \mathrm{~mm}$ scaffolds were secured with an intramedullary (IM) nail and 4 of the scaffolds were secured with plates. In 4 pigs a $40 \mathrm{~mm}$ osteotomy was created, filled with a $40 \mathrm{~mm}$ scaffold, and secured with plates. Fracture healing was assessed 3 months post-operatively using the Radiographic Union Score for Tibial Fractures (RUST) criteria.

\section{Results:}

For the $25 \mathrm{~mm}$ IM group, none of the 8 pigs achieved cortical bone union at 3 months post-op. In contrast, cortical union was observed in all of the $25 \mathrm{~mm}$ defects secured with plates. None of the $40 \mathrm{~mm}$ defects secured with plates achieved cortical union.

\section{Conclusion/Potential Impact:}

The failure of the $25 \mathrm{~mm}$ defect secured with an IM nail and the $40 \mathrm{~mm}$ defect secured with plates demonstrates that these are critical sized defect models. However, because the $25 \mathrm{~mm}$ defect secured with bridge plates did heal, we have termed this a metacritical sized defect. Identification of a defect size that can heal or not heal based on the fixation technique is a powerful translational model for testing therapies. 